\title{
Walter Kohn to Give Plenary Address at 2009 MRS Spring Meeting
}

Nobel laureate Walter Kohn of the University of California, Santa Barbara will give the plenary address at the 2009 Materials Research Society Spring Meeting to be held April 13-17 in San Francisco. The plenary session will be held Wednesday, April 15, at 5:30 p.m. in the San Francisco Marriott Hotel.

The search for renewables has become more urgent as people look for alternatives to fossil fuels. As part of the Meeting's WEDNESDAY SPOTLIGHT event, Kohn will discuss the vast and powerful potential of photovoltaics and solar energy for the developing world, including a screening of his spellbinding documentary film, The Power of the Sun. The documentary begins with the findings of Isaac Newton and other early visionaries, moving to the groundbreaking work in 1905 of Albert Einstein on photons, to Bell Laboratories in the 1950s, where the first silicon solar cell was produced.

The Power of the Sun provides insight into

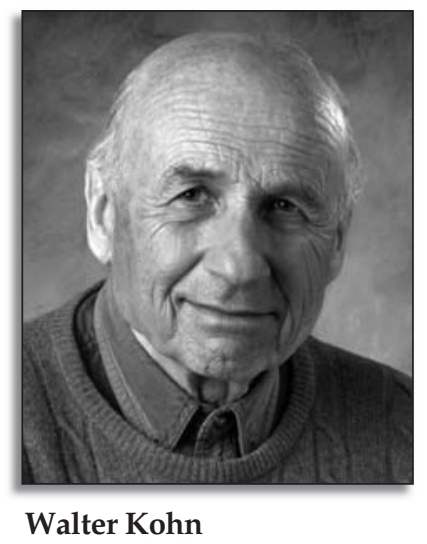

the clean logic of solar energy, its efficiency, and many applications. A major current challenge for materials research concerns interfaces (e.g., CdS/CdTe). Kohn worked with director/writer David Kennard and others to construct this optimistic and timely presentation. John Cleese of Monty Python fame serves as host and narrator of the documentary, making the material accessible for all audiences.

Kohn is a theorist on condensed matter who has made seminal contributions to the understanding of the electronic structure of materials. He played the leading role in the development of the density functional theory, which has revolutionized scientists' approach to the electronic structure of atoms, molecules, and solid materials in physics, chemistry, and materials science. He was awarded the Nobel Prize in Chemistry in 1998 for his development of density functional theory. Kohn has also made major contributions to the physics of semiconductors, superconductivity, surface physics, and catalysis. He was the founding director of the National Science Foundation's Institute for Theoretical Physics at the University of California, Santa Barbara, which brings together leading scientists from throughout the world to work on major problems in theoretical physics and related fields.

\section{Teri W. Odom Named 2009 MRS Outstanding Young Investigator for Nanophotonics}

Teri W. Odom, associate professor and the Dow Chemical Company Research Professor in the Department of Chemistry and the Department of Materials Science and Engineering at Northwestern University, has been named the 2009 Materials Research Society Outstanding Young Investigator. Odom was cited for "the development and characterization of nanoparticles and nanostructured arrays designed to filter and propagate plasmonic excitations with unprecedented control and sensitivity." She will deliver an award talk at the Materials Research Society Spring Meeting in San Francisco on April 14, at 9:30 a.m.

Odom's pioneering large-area nanoscale fabrication techniques have enabled investigations on nanoscale plasmonics. Her research group focuses on optical properties of nanohole arrays in metallic films, nano-pyramids, and multilayered nanoparticles; all reveal surprising phenomena. Using near-field scanning optical microscopy, Odom has imaged how surface plasmons form standing waves between nanoholes and how these waves can be manipulated by changing the material or the wavelength of the incident light. Combining near-field, far-field, and modeling techniques to investigate large-area nanohole arrays, Odom provided direct, conclusive evidence for the role of surface plasmons in the enhanced transmission.

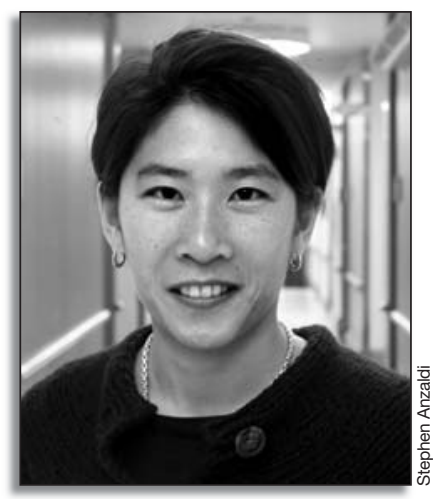

Teri W. Odom

Her collaborative work with theorists has given a conclusive set of linkages between the shapes and environments - of both nanoparticles and nanoholes-and their optical response.

Furthermore, Odom uses her unique methods to create devices for fundamental studies of the optical and plasmonic responses (experimentally and theoretically) with an eye toward applications in photonics and chemical sensing. Her fundamental studies combining quantum optics and materials synthesis are expected to lead toward developing new forms of materials that exhibit novel optical properties.

Odom received her PhD degree in chemical physics from Harvard in 2001, followed immediately by a postdoctoral fellowship there. She then began her tenure at Northwestern in 2002. Odom's many honors include, most recently, the NIH (National Institutes of Health) Director's Pioneer Award and the National Fresenius Award by Phi Lambda Upsilon and the American Chemical Society (ACS) in 2008; Rohm and Haas New Faculty Award (2007); the Exxon-Mobil Solid State Chemistry Faculty Fellowship from ACS Inorganic (2006); and in 2005, the Alfred P. Sloan Research Fellowship, the DuPont Young Investigator, and the Cottrell Scholar Award (Research Corporation). In 2004, she was named one of the world's 100 Top Young Innovators by MIT's Technology Review and prior to that she received a David and Lucile Packard Fellowship (2003).

Odom has more than 40 publications as well as over 20 predoctoral and postdoctoral articles, including articles in Nature, Science, and Advanced Materials. She has two U.S. patents and has served on advisory and editorial boards, including the Basic Energy Science Advisory Committee (BESAC) Grand Challenges Subcommittee for the Department of Energy, 2006-2008; the Japanese-American Kavli Frontiers of Science (JAFOS) Planning Group, 2008 and 2010; and was guest co-editor of a "Special Issue on Nanoscience" for Accounts of Chemical Research, published in December 2008. 